\title{
Perfilación geográfica del hurtador en Barcelona
}

\section{Geographical profiling of thiefs in Barcelona}

\section{Perfil geográfico do furtador em Barcelona}

\author{
Alba Pedrola Navarro *a $\mid$ Ariadna Trespaderne Dedeu ${ }^{b}$ \\ a https://orcid.org/0000-0002-4906-6313 Universidad de Barcelona, Barcelona, España

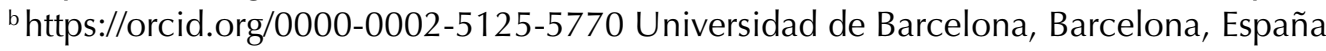

- Fecha de recepción: 2021-02-27

- Fecha concepto de evaluación: 2021-04-17

- Fecha de aprobación: 2021-04-29

https://doi.org/10.22335/rlct.v13i2.1423
Para citar este artículo / To reference this article / Para citar este artigo: Pedrola, A., \& Trespaderne, A. (2021). Perfilación geográfica del hurtador en Barcelona. Revista Logos Ciencia \& Tecnología, 13(2), 115-133. https://doi.org/10.22335/rlct.v13i2.1423

\section{RESUMEN}

El hurto es el delito más recurrente en Barcelona, que causa una percepción de inseguridad ciudadana, y que plantea la necesidad de crear estrategias de prevención e intervención por parte de la policía, siendo de gran ayuda la perfilación geográfica. Así, el objetivo principal de esta investigación es establecer el perfil geoespacial de una muestra de hurtadores, mediante un estudio empírico, descriptivo y aplicado, en el que se empleó el método cuantitativo. Se partió de una muestra inicial de 66 delincuentes, en la cual se analizó la distancia del domicilio al lugar del hurto, y de una segunda muestra de 15, para comparar los perfiles de los commuters y los maraduers y establecer la safety zone y la decay distance. De acuerdo con la teoría; los ladrones nocturnos viven más cerca del lugar del hurto que los diurnos, la mayoría residen en la zona metropolitana y hurtan aproximadamente a unos $5 \mathrm{~km}$ de su domicilio. Se distribuyen equitativamente entre commuters y marauders, siendo los marauders los que recorren distancias más cortas y delinquen de forma más recurrente. Concretamente, en Barcelona, tres de cada diez vuelven a delinquir dentro de los 70 días posteriores al hurto; para los marauders se estableció la safety zone a una distancia de $0.36 \mathrm{~km}$ y la decay distance a $3 \mathrm{~km}$ de su domicilio.

Palabras clave: commuters, distancia, hurto, marauders, policía 


\begin{abstract}
Theft is the most recurrent crime in Barcelona and causes a perception of public insecurity that generates the need to create prevention and intervention strategies by the police, being of great help the geographic profile. The main objective of this research is to establish the geospatial profile of a sample of thieves, through an empirical, descriptive and applied study, in which the quantitative method is used. It started from an initial sample of 66 shoplifters to analyze the characteristics of the distances from home to theft, and a second sample of 15 to analyze the characterization of commuters vs. marauders, as well as the safety zone and the decay distance. Coinciding with the theory, night robbers live closer to the place of theft than daytime robbers, most of them reside in the metropolitan area and steal about $5 \mathrm{~km}$ from their home; they are distributed equally between commuters and marauders, traveling the latter shorter distances and committing offenses on a recurring basis. Specifically in Barcelona, three out of ten robs within 70 days after the theft, and the marauders, between a range located $0.36 \mathrm{~km}$ from the home (safety zone) up to approximately $3 \mathrm{~km}$ (decay distance).
\end{abstract}

Keywords: commuters, distance, marauders, police, theft

\title{
RESUMO
}

O furto é o crime mais recorrente em Barcelona, o que provoca uma percepção de insegurança nos cidadãos e que suscita a necessidade de criar estratégias de prevenção e intervenção por parte da polícia, sendo o perfil geográfico de grande ajuda. Assim, o objetivo principal desta pesquisa é estabelecer o perfil geo-espacial de uma amostra de ladrões, por meio de um estudo empírico, descritivo e aplicado, no qual foi utilizado o método quantitativo. Partiu-se de uma amostra inicial de 66 criminosos, na qual foi analisada a distância de casa ao local do furto, e de uma segunda amostra de 15, para comparar os perfis de passageiros e saqueadores e estabelecer a zona de segurança e de decadência. De acordo com a teoria; os ladrões noturnos vivem mais perto do local do furto do que os ladrões diurnos, a maioria deles reside na área metropolitana e rouba em torno de $5 \mathrm{~km}$ de sua casa. Eles são distribuídos igualmente entre passageiros e saqueadores, sendo os saqueadores aqueles que viajam as distâncias mais curtas e cometem crimes com mais frequência. Especificamente, em Barcelona, três em cada dez cometem crimes novamente dentro de 70 dias após o furto; para os saqueadores, a zona de segurança foi estabelecida a uma distância de $0,36 \mathrm{~km}$ e a distância de decadência a $3 \mathrm{~km}$ de sua casa.

Palavras-chave: passageiros, distância, furto, saqueadores, polícia

Durante los últimos años, en la ciudad de Barcelona, España, el hurto se ha convertido en el delito con más denuncias, lo cual ha provocado una gran percepción de inseguridad por parte de la población. En consecuencia, la preocupación de los políticos y de las fuerzas de seguridad por mejorar sus esfuerzos para garantizar la seguridad ciudadana ha aumentado.

Cabe señalar que, a pesar de su incremento, el hurto no es un delito contra las personas y no emplea la fuerza, la violencia o la intimidación, razón por lo cual recibe menos atención que el robo, en el que sí existe fuerza, violencia o intimidación; no obstante, en ambos delitos el objetivo del delincuente es apoderarse de los bienes ajenos.
Sin embargo, el impacto del hurto sobre la percepción de inseguridad ciudadana es alto, ya que se produce de forma muy recurrente. Esto repercute enormemente tanto en la población residente como en el turista, a la hora de elegir su destino. Es por ello que organismos nacionales e internacionales como la ONU buscan implementar medidas, técnicas, estrategias y nuevas herramientas para contrarrestar estos hechos delictivos.

Para reducir esta alta incidencia de hurtos, se plantean un conjunto de técnicas y herramientas encaminadas a identificar y localizar a los ladrones. Entre ellas, la perfilación geográfica se configura como una técnica capaz de localizar el área en la que existe mayor probabilidad de encontrar al delincuente, hecho que facilita su deten- 
ción y disminuye la frecuencia de hurtos, consiguiendo, además, reducir el número de efectivos policiales y facilitando que se resuelvan estas investigaciones con más rapidez y eficacia.

Por todos estos aspectos, se considera de suma importancia analizar las técnicas que pueden reducir la incidencia del hurto en Barcelona, un delito cada vez más recurrente, que no recibe la atención que merece y frente al cual las fuerzas y cuerpos de seguridad deben emplear recursos de la forma más eficiente.

\section{El hurto}

El hurto en España es categorizado como delito contra el patrimonio y el orden socioeconómico, que afecta directamente a la ciudadanía debido a la disminución directa de su capital. Se encuentra regulado en el artículo 234 del Código Penal Español (Jefatura del Estado Español, 1995) en dos modalidades: por cuantías superiores a $€ 400$ y por cuantías inferiores a esta (hurto leve); ambos delitos son penalizados con una condena de prisión de 6 a 18 meses y de 1 a 3 meses, respectivamente.

El hurto leve es denunciado a diario a la policía local de Barcelona (en adelante GUB), debido a que los hurtadores, conscientes de su escasa repercusión, optan por apropiarse de objetos ajenos, menos valiosos, aunque de forma más recurrente, convirtiéndose en uno de los principales delitos que inciden en la percepción de inseguridad ciudadana (Bernasco, Johnson \& Ruiter, 2015; Goodwill \& Alison, 2006; Long et al., 2018).

Por ello, las investigaciones criminológicas actuales apuestan hacia un modelo basado en la prevención y no tanto por un modelo punitivo (Bowers \& Johnson, 2016). Dentro de los diferentes ámbitos desde los que se puede enfocar la prevención (Cuerpos y Fuerzas de Seguridad, desde el entorno social, la observación de los autores y el diseño ambiental), la presente investigación está orientada, desde la estrategia policial, a la creación de un perfil geográfico criminal. Con esto se busca establecer patrones geoespaciales y comportamentales del hurtador, ya que se considera que este comete sus delitos en zonas repartidas de forma irregular sobre el territorio (Lantz \& Ruback, 2017), siguiendo un patrón de frecuencia en algunas de ellas (Eck, 2018; Weisburd, 2015), distribuidas con cierta lógica espacial (Felson \& Boivin, 2015). Estas precisiones permiten considerar este delito no como producto de la oportunidad y el azar, además de proporcionar información útil para aplicar tácticas preventivas y de actuación en ciertos puntos de la ciudad.
El presente trabajo sustenta su modelo teórico en diversas teorías criminológicas encuadradas dentro de la vertiente denominada criminología ambiental, centrada en la distribución espacial y temporal de los hechos delictivos, ya que, para poder llevar a cabo el hurto con éxito, este debe cometerse en el sitio y la hora apropiados, teniendo en cuenta que no todos los sitios son idóneos para lograr los objetivos y que estos variarán según el día y la hora de la semana (Bernasco, Ruiter \& Block, 2017; Haberman \& Ratcliffe, 2015; Hipp \& Kim, 2019; Sidebottom \& Wortley, 2016). Las teorías que se exponen a continuación ofrecen explicaciones a nivel macro sobre las variaciones espaciotemporales de la delincuencia (y también del hurto), en contextos ambientales particulares (Hart \& Miethe, 2014), y ayudan a entender los factores influyentes en la toma de decisiones del hurtador.

\section{Teorías criminológicas}

La teoría de la elección racional de Cornish \& Clarke (1986) postula que el delito es el resultado de una decisión razonada por parte del infractor, en la cual se hace una valoración sobre los costes y beneficios del delito; en este sentido, la decisión de actuar se toma en función de los obstáculos y las oportunidades del momento y el lugar, eligiendo los objetivos y las ubicaciones que maximicen los beneficios y reduzcan los riesgos (Bernasco \& Nieuwbeerta, 2005; Cornish \& Clarke, 1986; Frith, Johnson \& Fry, 2017).

Por otra parte, la teoría de las actividades rutinarias de Cohen \& Felson (1979) indica los factores necesarios para facilitar el delito: la convergencia en el espacio y el tiempo de un delincuente con un objetivo, una posible víctima y la falta de vigilancia (Bernasco et al., 2017); la ausencia de uno de estos factores tiene como resultado el aplazamiento o la omisión del hecho delictivo (Branic, 2015). Por el contrario, la delincuencia tiene lugar en los espacios donde todos estos factores desencadenantes están presentes (Boivin, 2018).

Por su parte, la teoría de la geografía del tiempo (Hägerstrand, 1989) sintetiza las restricciones que afectan a las actividades individuales en el espacio y el tiempo (Bernasco et al., 2017; Tucker et. al., 2021). Por un lado, las restricciones de capacidad de los individuos incluyen restricciones biológicas (e.g., la necesidad de dormir) y limitaciones tecnológicas como la falta de transporte) (Piza \& Kennedy, 2003). Por otro lado, las restricciones de acoplamiento requieren que las personas estén en lugares y momentos específicos por los roles sociales que cumplen, (e.g., el horario escolar o de trabajo demandan que las 
personas estén en la escuela o en el lugar de trabajo). Y, por último, las restricciones de las autoridades limitan el acceso a ciertos lugares (e.g., en los centros comerciales en horario nocturno) o los propietarios de algunos sitios solo permiten la entrada a determinados individuos y la bloquean para otros (Bernasco et al., 2017; Monk, Heinonen \& Eck, 2010). En definitiva, las restricciones limitan la libertad de las personas para decidir dónde y cuándo estar. Por lo tanto, al abordar los aspectos temporales para la elección de la ubicación delictiva es importante tener en cuenta que tanto los delincuentes como las víctimas están sometidos a estas restricciones. Pero además de esto, Bowers \& Johnson (2016) muestran que la existencia de ciertas necesidades, como la de dormir, conllevan a que el hurto se cometa en zonas más cercanas al domicilio durante las horas en que la gente se despierta (7:00-9:00) y se acuesta (entre las 22:00 y la medianoche) (Vandeviver \& Bernasco, 2019), y que estos delitos sucedan más lejos fuera de estas horas (Bernasco et al., 2017). También se entiende que durante el fin de semana se cometan los hurtos a una distancia más lejana al domicilio que entre semana, ya que las obligaciones en días laborales hacen que las personas se desplacen a lugares más próximos al domicilio y lugar de trabajo, en comparación del fin de semana (Bowers \& Johnson, 2015).

La teoría del patrón delictivo (Brantingham y Brantingham, 1981; 2008) analiza específicamente los lugares donde los delincuentes realizan sus actividades $y$ establece que existen dos condiciones necesarias para que ocurra un delito en un lugar determinado: el lugar debe proporcionar una oportunidad para que se produzca la comisión del delito y el posible delincuente debe ser consciente del lugar y la oportunidad (Bernasco et al., 2017). Además, esta teoría señala que los infractores cometen sus delitos cerca de su domicilio, es decir, no se desplazan lejos para delinquir (Bernasco et al., 2015; Phillips, 1980; Rengert, Piquero \& Jones, 1999; Rhodes \& Canly, 1981; Sarangi \& Youngs, 2006), porque conocen más la zona y desplazarse más lejos les cuesta más tiempo y esfuerzo (Pettiway, 1982). Del mismo modo, también existen más probabilidades de delinquir en zonas cercanas a los lugares donde los hurtadores desempeñan sus actividades rutinarias, como el colegio o el trabajo (Bernasco, Block \& Ruiter, 2013).

Perfil geográfico del hurtador

La mayoría de criminales delinquen en una zona delimitada denominada rango criminal. Esta zona se encuentra entre la llamada safety zone (Canter \& Hodge, 2000), es decir, el área que se encuentra cerca de su domicilio, y en la que no roban para no ser reconocidos, y la decay distance (Rossmo, 1995), o distancia máxima donde dejan de delinquir. También Canter \& Larkin (1993) crearon una clasificación del perfil criminal en función de la distancia recorrida desde el domicilio hasta el lugar donde suceden los hechos, distinguiendo entre commuters y marauders, siendo los primeros los que recorren mayor distancia para la comisión del delito y los últimos los que cometen el delito más cerca del domicilio. Así, los commuters se caracterizan por buscar zonas alejadas de su domicilio para delinquir, y los marauders se desplazan en múltiples direcciones, priorizando la proximidad. Este patrón geográfico de comportamiento resulta en una diferenciación muy operativa: el domicilio de los commuters se encuentra fuera del radio creado entre los dos delitos más lejanos, mientras que el de los marauders se halla dentro. En consecuencia, la safety zone solo se puede concretar para los marauders; en cambio, la decay distance se puede definir para ambos tipos, aunque solo se cumpla estrictamente entre los marauders (Kocsis \& Irwin, 1997).

La decay distance de los marauders puede ser representada por una curva (Rossmo, 1995) que indica cómo la probabilidad de cometer el delito disminuye a medida que la distancia desde su ubicación residencial aumenta (Bernasco, 2007; Bernasco \& Kooistra, 2010; Bernasco et al., 2015; Canter \& Larkin, 1993; Emeno \& Bennell, 2013; Kocsis \& Irwin, 1997; Sarangi \& Youngs, 2006). Aunque esta también se podría aplicar a los commuters, solo se adecúa estrictamente a los marauders (Emeno \& Bennell, 2013; Kocsis \& Irwin, 1997). En cambio, la direccionalidad en la comisión del delito tiene más sentido de ser analizada en el caso de los commuters, ya que presentan una tendencia más marcada a delinquir en una única dirección, mientras que los marauders son más multidireccionales.

La aplicación de la teoría del círculo de Canter (Canter \& Larkins, 1993) permite realizar una mejor distinción entre commuters y marauders. Plantea la existencia de una base fija (punto de anclaje) desde donde viaja el delincuente para hurtar dentro de un área determinada. La teoría se creó para descubrir dónde se sitúa el punto de anclaje de un delincuente serial que haya cometido más de cinco delitos relacionados entre sí; consiste en unir mediante una línea los dos delitos más alejados y, tomando esta línea como diámetro, trazar un círculo de forma que encierre al resto de delitos. Aunque la teoría se planteó inicialmente para asesinos seriales, se aplica actualmente a otras tipologías delictivas, entre ellas el hurto. Existen así investigaciones que han intentado determinar si hay mayor porcentaje de commuters o ma- 
rauders entre los delincuentes. Bernasco et al. (2015) defienden que el perfil de ambos está distribuido de forma equitativa. No obstante, otros autores como Lantz \& Hutchison (2015) toman en cuenta otros criterios, como la frecuencia temporal, a la hora de analizar perfiles.

A partir de la aplicación de la teoría del círculo de Canter al hurto, tal como se acomete en la presente investigación, se puede analizar el rango criminal (zona donde se cometen los delitos); la distancia entre el domicilio del hurtador y el delito más lejano, definida como la distancia más larga que un delincuente está dispuesto a viajar con tal de cometer el delito (Canter \& Larkins, 1993); el perfil del hurtador (commuter o marauder) y, de clasificarse como marauder, también se puede determinar la safety zone; la direccionalidad, la distance decay y el patrón delictivo.

\section{Objetivos}

\section{Objetivo general}

Establecer el perfil geoespacial de una muestra de hurtadores de Barcelona.

\section{Objetivos específicos}

1. Analizar la distancia recorrida entre el domicilio del hurtador y el hurto, y si existe una relación de dependencia entre esta, el sitio, la hora y el día.

2. Describir, a partir de los antecedentes, el tipo de hurtador (marauder o commuter), la distancia media recorrida, la frecuencia temporal, y si existe dependencia entre ellas.

3. Determinar la safety zone en los marauders de Barcelona, y si estos presentan una direccionalidad privilegiada en la comisión del hurto.

4. Representar la decay distance de los hurtadores en Barcelona.

\section{Metodología}

\section{Diseño de investigación}

La investigación es un estudio empírico-descriptivo, ya que las conclusiones se extraen estrictamente de pruebas empíricas, concretas y verificables, que parten de una muestra extraída de una fuente primaria. Es de tipo descriptivo, porque busca conocer cómo se manifiesta geográficamente el hurto en la ciudad de Barcelona y especificar las características geoespaciales de los hurtadores. Además, posee una finalidad aplicada, pues pretende obtener una explica- ción que pueda ser útil para la GUB, con tal de mejorar la estrategia policial y aumentar su efectividad.

Es un diseño observacional, porque trata de describir un fenómeno dentro de una población de estudio y conocer su distribución. No existe ninguna intervención por parte del investigador, sino que se limita más bien a medir el fenómeno y a describirlo tal y como se encuentra en la población de estudio. Así, su observación es indirecta, debido a que se realiza a través de las observaciones realizadas por agentes de la GUB, encargados de recopilar los datos facilitados y explotados para la presente investigación, no siendo la investigadora la encargada de recolectar los datos.

El estudio no es experimental, porque no se controlan ni manipulan las variables del estudio, sino que los hechos ya sucedieron. Las variables de los hurtos cometidos en Barcelona fueron recogidas por los agentes de la GUB, quienes han presenciado el delito o han recolectado los hechos descritos por las propias víctimas. Desde el punto de vista espaciotemporal, el estudio se clasifica como transversal, debido a que se realiza durante las dos primeras semanas de marzo con la finalidad de describir variables, analizar su incidencia e interrelación en un momento concreto.

Por último, el método es cuantitativo; es decir, se centra en aspectos observables susceptibles de cuantificación y utiliza la estadística para el análisis de los datos, con la finalidad de ponderar el problema y entender su grado de generalización.

\section{Muestra}

El presente estudio contó con una muestra inicial de 71 delincuentes que habían cometido 56 hurtos en la ciudad de Barcelona, entre el 1 y el 10 de marzo de 2020. Esta información se obtuvo de los atestados de la GUB recogidos durante estas fechas. Se trata finalmente de una muestra no probabilística, por conveniencia y no aleatoria.

Para cumplir el primer objetivo, se analizó la distancia recorrida entre el domicilio del hurtador y el lugar del delito, y si existía una relación de dependencia entre este, el sitio y la hora. Se excluyeron cinco casos de la muestra, porque en los atestados no se recogía la ubicación del domicilio del sujeto; es decir, se realizó este análisis con la información de 66 infractores.

Para el segundo objetivo, se caracterizó el tipo de perfil (marauder o commuter), a partir de los antecedentes. Se 
determinó la distancia media recorrida, la frecuencia temporal y la existencia de una dependencia entre ellas. Para ello, se empleó una muestra de 15 casos, que aplicaron dos criterios de exclusión. Según el primer criterio, se excluyeron los hurtadores que no poseían más de 4 antecedentes, porque según la teoría de Canter \& Larkins (1993), esto es un requisito sine qua non para poder aplicar la teoría del círculo de Canter, lo que resultó en la exclusión de 29 casos. Según el segundo criterio, se excluyeron los delincuentes cuyos antecedentes no eran delitos económicos o contra la propiedad (22 casos), pues para este estudio lo que interesa es el análisis del perfil del ladrón, sin entrar en otros posibles delitos que este haya podido ocasionar. En definitiva, después de aplicar los dos criterios de exclusión, se partió de una muestra de 15 sujetos.

Para el tercer objetivo, se analizó la safety zone de los marauders y si estos presentan una dirección privilegiada. De los 15 casos seleccionados para el análisis del tercer objetivo, se eliminaron los casos de los commuters, ya que los hurtadores debían ser marauders, como criterio indispensable para garantizar la precisión de los resultados. De este modo, tras descartar 7 casos, la muestra final fue de 8 perfiles de marauders.

Finalmente, para el análisis de la decay distance, que constituye el cuarto objetivo de este trabajo, se utilizó la muestra de 15 casos detallada en el segundo párrafo de este apartado. Esta muestra se distribuyó en dos grupos: commuters ( 7 casos) y marauders (8 casos), y también se analizaron los 15 sujetos en conjunto (grupo mixto).

\section{Instrumentos}

La base de datos utilizada a lo largo de esta investigación fue creada durante las prácticas universitarias del Máster de Perfilación y Análisis de la Conducta Criminal de la Universidad de Barcelona, en la Unidad de Investigación de la Guardia Urbana de Barcelona (UIGUB). Se recopilaron los atestados que Ilegaron a dicha unidad desde el 1 hasta el 10 de marzo de 2020, provenientes de los distintos distritos que integran la ciudad de Barcelona, facilitados por la UIGUB en formato papel.

Para hacer el vaciado de información se utilizó el programa Excel, con el fin de registrar la información relativa a los hurtadores de Barcelona: fechas, ubicaciones, residencias y antecedentes.

\section{Selección de las variables}

En la creación de este instrumento de recogida de datos se tuvieron en consideración las características geográficas de los hurtos, así como el perfil de los delincuentes y sus antecedentes penales (tabla 1).

Tabla 1

Variables utilizadas en el instrumento

\begin{tabular}{cl}
\hline \multicolumn{1}{|c|}{ Dimensiones } & \multicolumn{1}{c}{ Variables } \\
& - Zona turística/no turística (turística: Ciutat Vella, Eixample i Gràcia; no turística: \\
Características geográficas & Sant Andreu, Sant Martí, Sants-Montjuic). \\
de los hurtos & - Comercio/no comercio (comercio y no comercio: superficie y transporte público). \\
& - Día/noche (claridad/oscuridad) \\
& - Parte de la semana (entre semana, fin de semana) \\
Características geográficas & - Franja de distancia entre el domicilio y el lugar del delito en km. \\
de los hurtadores & - Tipo de distancia (corta/larga) \\
& - Tipo de delincuente (commuter/marauder) \\
Características de & - Distancia recorrida \\
los antecedentes (delitos & - Tipo de distancia (corta/larga) \\
económicos anteriores) & - Frecuencia (alta/baja) \\
& - Safety zone \\
\hline
\end{tabular}

Nota: Elaboración propia a partir de la información extraída de los atestados de la GUB.

Las anteriores variables han sido categorizadas de la siguiente forma:

1. Características geográficas de los hurtos
- Zona turística/no turística: según la clasificación de Brunt \& Hambly (1999), se categoriza el centro de la ciudad como zona turística y las zonas periféricas como no turísticas. Así, se clasificó la Ciutat Ve- 
Ila, Eixample y Gràcia como zonas turísticas; y Sant Andreu, Sant Martí y Sants Montjuic como zonas no turísticas.

- Comercio o no comercio: se clasificaron los hurtos en función de si fueron cometidos en un comercio o no.

- Hora: hora concreta (considerando horas enteras) en las que se cometió el hurto.

- Luz u oscuridad: esta clasificación se estableció en función de si todavía hay luz solar o no (claridad/oscuridad) en el momento del delito; esta clasificación la utilizaron Tompson \& Bowers (2013) para definir si la oscuridad influye en el número de hurtos, o si por el contrario se cometen más delitos durante el día. Para ello, se han considerado cuáles eran las horas de sol durante las fechas que se recogió la muestra (entre el 1 y el 10 de marzo del 2020), resultando ser las horas de sol las comprendidas entre las 6:00 y las 18:00 horas $y$, el resto horas sin luz natural (oscuridad).

- Parte de la semana: los casos se agrupan en función de si el hurto se cometió entre semana o el fin de semana, clasificación recogida por Glasner \& Leitner (2017).

2. Características geográficas del hurtador

- Distancia del domicilio del autor al lugar del hurto: se calculó en kilómetros enteros la distancia que recorrió el ladrón desde su domicilio hasta el lugar del hurto.

- Tipo de distancia del domicilio del autor al lugar hurto: se estableció la categoría de distancia como corta o larga, a partir del cálculo de la media aritmética de la distancia recorrida por los delincuentes hasta el lugar donde cometieron los robos; en este caso, la media fue de $5.92 \mathrm{~km}$. Esta distancia marcó el punto intermedio entre las distancias recorridas en cada caso: se clasificó como corta toda aquella distancia que quedó por debajo de la media, y como larga toda distancia que estuvo por encima.

3. Antecedentes del hurtador

- Tipo: commuter o marauder, siguiendo la clasificación que realiza Canter \& Larkin (1993).

- Distancia recorrida: número de kilómetros que recorrió el infractor desde su domicilio hasta el lugar donde cometió el hurto.

- Media recorrida: se realizó la media aritmética entre las distancias que recorrió cada delincuente hasta llegar a cada uno de los sitios donde se cometió el delito principal y los anteriores (mínimo 4).

- Tipo de distancia: corta o larga, se calculó realizando la media aritmética de las medias calculadas en el apartado anterior (media recorrida), las medias recorridas del domicilio del hurtador hacia cada uno de sus robos anteriores y al hurto principal. De cada una de estas medias se calculó nuevamente la media aritmética, dando un resultado de $8.62 \mathrm{~km}$. Esta distancia se estableció como punto medio, es decir que las distancias que se encuentran por encima de la media son largas y las que están por debajo son cortas.

- Frecuencia: media de frecuencia entre los delitos cometidos por cada delincuente y la media de las frecuencias entre todos ellos. Por encima de la media (71 días) se consideró baja frecuencia; por debajo de esta, alta frecuencia.

También se calculó la safety zone, utilizando el mismo método que se recoge en el artículo de Kocsis \& Irwin (1997), y la decay distance, utilizando el método recogido en el artículo de Block \& Bernasco (2009).

Análisis de datos

En la presente investigación se utilizaron distintos programas: inicialmente, un Sistema de Información Geográfica (QGIS 3.8), que permitió llevar a cabo un primer análisis descriptivo sobre la muestra de criminales, presentado en forma de crime mappings: el primero (figura 1) permite situar dónde suceden los hurtos y dónde residen los 66 delincuentes; el segundo (figura 2) muestra la direccionalidad y el trayecto que sigue el ladrón desde su domicilio hasta el lugar donde comete el hurto, seguido de otros (figuras 4 y 5 ) que muestran el lugar de residencia de los delincuentes, dónde actúan los nocturnos y los diurnos, y los que actúan entre semana y el fin de semana (figuras 6 y 7). Luego viene otro mapping (figura 8) que muestra los lugares de comisión del hurto principal, los de los anteriores y el domicilio del hurtador (de los 15 seleccionados según criterios de inclusión), y, por último, aparecen dos mapas de redes (figuras 9 y 10) que permiten observar la direccionalidad, la zona de procedencia del hurtador y la de comisión del hurto. Sin embargo, previo a la creación de estos mapas se han geolocalizado los domicilios, el lugar de los delitos y se calcularon las distancias recorridas mediante el programa Google Maps. Toda la información se incorporó en una tabla Excel, para posteriormente ser explotada con el QGIS.

El segundo programa que se utilizó fue el IBM SPSS Statistics Program, versión 22.0, mediante el que se realizó un análisis descriptivo, expuesto en forma de tablas, sobre el tipo de distancia recorrida del domicilio del asaltante al lugar donde comete el hurto (tabla 2), el tipo de hurtador, la distancia recorrida, el tipo de distancia recorrida y la frecuencia temporal (tabla 7). 
Además, el SPSS se utilizó para llevar a cabo un modelo de análisis bivariado y de significancia mediante prueba de Chi cuadrado, que permitió identificar si existe asociación entre las siguientes variables: tipo de distancia en relación con la zona (tabla 3), tipo de distancia relacionada con la luz del día (tabla 4), tipo de distancia relacionada con el sitio turístico/no turístico (tabla 5) y tipo de distancia en relación con la parte de la semana en que se realiza el robo (tabla 6).

Para medir el tamaño del efecto del Chi cuadrado se utilizó la escala de Cohen (citado en Castro \& Martini, 2014). Esta escala va de 0 a 1 : no efecto $(0-0.10)$, efecto pequeño (0.10 - 0.30), efecto mediano o moderado (0.30 - 0.50), efecto grande (0.50 - 1.00). Finalmente, se aplica $\vee$ de Cramer para los resultados que presentan significación.

Para poder ubicar a un infractor dentro de la tipología de commuter o marauder se utilizó el programa CrimeStat Spatial Statistics Program (ICPSR), versión 3.0, para representar los resultados posteriormente en el círculo de Canter del QGIS y así determinar dentro de cuál tipología se encuentra cada sujeto de la muestra. Además, utilizando el SPSS se llevaron a cabo las tablas de contingencia para observar la distribución de la muestra de: commuters/ marauders, respecto a la frecuencia temporal (tabla 8); commuters/marauders, respecto a la distancia recorrida (tabla 9) y, finalmente, el tipo de distancia, respecto a la frecuencia temporal (tabla 10).

Teniendo en cuenta únicamente la información de los marauders, y utilizando el mismo método que se recoge en el artículo de Kocsis \& Irwin (1997), se calculó la safety zone y se analizó si estos robos presentaron una direccionalidad privilegiada. Este análisis consiste en representar para cada marauder, de los cuales se conocen los antecedentes, la distancia máxima entre sus delitos, en función de la distancia de su domicilio y el hurto más lejano realizado, para aplicar una regresión lineal $(y=a x+b y=a x+b)$ (figura 11).

El valor de la pendiente $(a)$, para los marauders, debe tomar valores entre 0.5 y 1 ; si resulta más próximo a 0.5 , como en los resultados (0.65), implica que el domicilio está centrado respecto a los hurtos cometidos, mientras que si es cercano 1 significa que el domicilio se encuentra en una posición excéntrica, es decir, descentrada respeto la distribución de los delitos. Por este motivo, identificamos este parámetro como el que determina si los sujetos de nuestra muestra, en promedio, presentan una direccionalidad respeto al domicilio a la hora de robar o si por el contario hurtan en todas direcciones por igual.
El término independiente en la recta de regresión (b) se interpreta como la existencia de una distancia mayor que cero, desde el domicilio hasta el lugar del hurto, incluso cuando se aplica el límite en el que se sitúan todos los delitos a una distancia nula entre ellos. El hecho de encontrar un valor de $b$ mayor que cero, como el de los resultados (0.36), implica la aparición de una safety zone alrededor del domicilio, es decir, en la zona en la que el hurtador no delinque.

Además, resulta indispensable, para que el resultado sea significativo, que el coeficiente de correlación de la regresión sea próximo a 1 ; en este caso, el resultado es de un valor de 0.98 .

Siguiendo el mismo modelo que tienen las investigaciones de Block \& Bernasco (2009) se analizó la distance decay, es decir el patrón que siguen los delincuentes a la hora de cometer el hurto, respecto a la distancia entre su domicilio y el lugar del delito, para los tres grupos: marauders, commuters y grupo mixto (figuras 12A, 12B y $12 \mathrm{C}$, respectivamente). Para llevar a cabo tal análisis, se presentó en un histograma de frecuencias la distancia recorrida del domicilio al lugar del hurto. Para el grupo de los marauders se aplicó una distribución exponencial negativa $y=A e-B x$ para modelizar el comportamiento en la distance decay (figura 13).

\section{Resultados}

\section{Delincuentes \\ Análisis descriptivo}

De los 66 sujetos de la muestra, la mayoría reside en el área metropolitana de Barcelona, en la zona donde se produce el mayor número de hurtos (distrito de Ciutat Vella), mientras que el resto se sitúa mayoritariamente en la zona de Sant Andreu- Sant Adrià- La Trinitat Vella- Badalona o en la zona de Hospitalet de Llobregat, y una pequeña proporción reside en afueras de Barcelona (figura 1).

Los delincuentes se desplazan hacia el centro para hurtar. Algunos recorren distancias más largas que otros, y unos pocos provienen de las afueras de Barcelona: su domicilio no es visible en el mapa, pero puede intuirse por las líneas procedentes de un área exterior (figura 2).

La mayoría de ladrones de Barcelona actúan cerca del domicilio; recorren una distancia entre 0 y $5 \mathrm{~km} \mathrm{y}$, a medida que se alejan, roban cada vez menos (figura 3 ). 
Figura 1

Hurtos y domicilio de los hurtadores

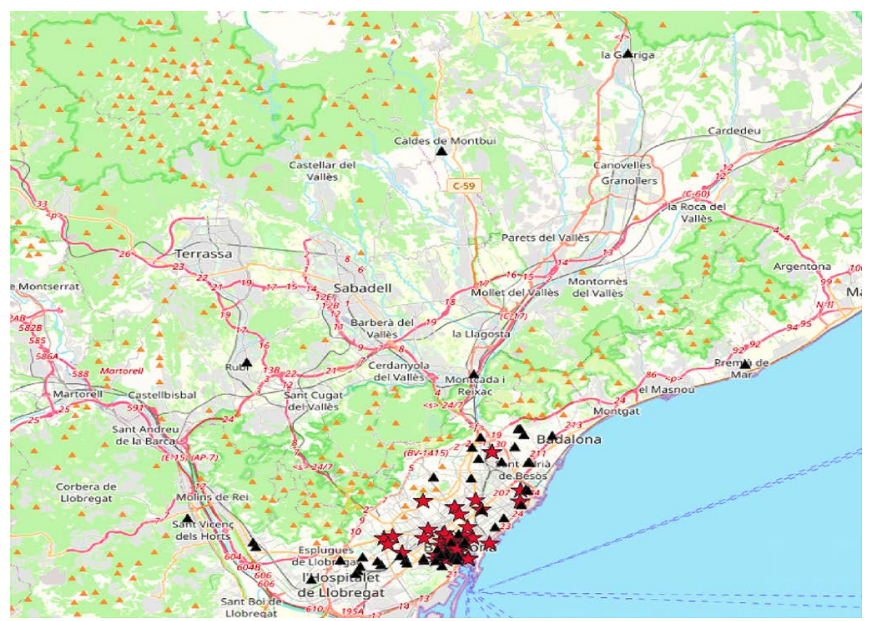

Nota:

$\mathbf{\Delta}$, domicilios de los hurtadores

$\hat{\imath}$, lugar donde se cometieron los hurtos.
Figura 2

Dirección del domicilio al hurto

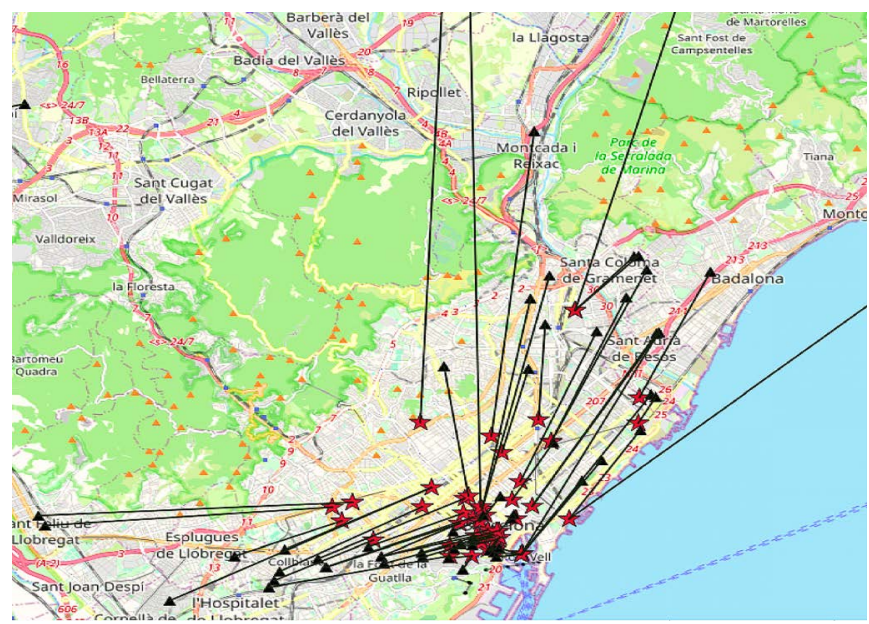

Nota:

$\mathbf{\Delta}$, domicilios de los hurtadores

得, lugar donde se cometieron los hurtos

$\longrightarrow$ recorridos y direccionalidad de los hurtadores.

Figura 3

Distancia recorrida desde el domicilio del delincuente hasta el lugar del hurto

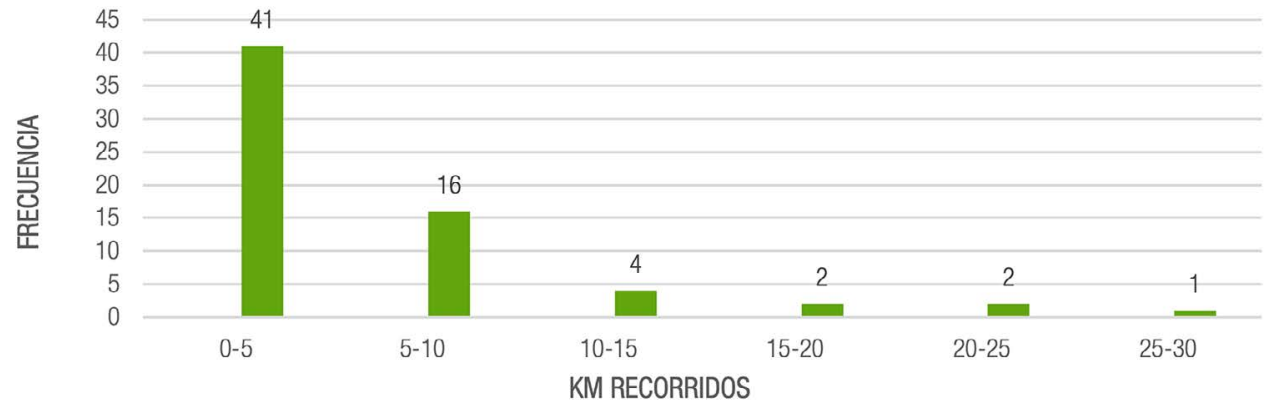

La mayoría de hurtadores recorren una distancia corta desde su domicilio al hurto hasta el lugar del hurto (tabla 2).

Tabla 2

Tipo de distancia recorrida desde el domicilio del ladrón hasta el lugar del hurto

\begin{tabular}{ccc}
\hline Tipo de distancia & Número & Porcentaje \\
\hline Corta $(<8.62 \mathrm{~km})$ & 42 & $63.6 \%$ \\
\hline Larga $(>8.62 \mathrm{~km})$ & 24 & $36.4 \%$ \\
\hline Total & 66 & $100 \%$ \\
\hline
\end{tabular}

Nota: Elaboración propia a partir de la información extraída de los atestados de la GUB.

\section{Análisis bivariado de los delincuentes}

El análisis del lugar de los hurtos muestra que estos están equitativamente repartidos entre el comercio y los espacios no comerciales, aunque se observa que para delin- quir en los comercios los ladrones recorren distancias más largas. La diferencia es estadísticamente significativa $\left(X^{2}(1)=17.569 ; p=0.001 ; V=0.001\right)$ (tabla 3$)$.

Tabla 3

Tipo de distancia (corta/larga) en relación con la zona (comercio o no comercio).

\begin{tabular}{ccccc}
\hline $\mathbf{n}=66$ & Comercio & No comercio & N & P \\
\hline Corta & 19 & 23 & 42 & \\
\hline Larga & 14 & 10 & 24 & $\mathrm{p}=0.001$ \\
\hline Total & 33 & 33 & 66 & \\
\hline
\end{tabular}

Nota: Elaboración propia a partir de la información extraída de los atestados de la GUB.

Los hurtos producidos en la oscuridad se realizan a poca distancia del domicilio, mientras que los realizados a plena luz del día se hacen a una mayor distancia. La di- 
ferencia es estadísticamente significativa $\left(X^{2}(1)=4.036\right.$; $\mathrm{p}=0.039 ; \mathrm{V}=0.045)$ y figuras 4 y 5 .

Tabla 4

Tipo de distancia (corta o larga) relacionada con las condiciones de luz (luz u oscuridad).

\begin{tabular}{ccccc}
$\mathbf{n}=\mathbf{6 6}$ & Luz & Oscuridad & N & p \\
\hline Corta & 19 & 23 & 42 & \\
Larga & 17 & 7 & 24 & $\mathrm{p}=0.039$ \\
Total & 36 & 30 & 66 & \\
\hline
\end{tabular}

Nota: Elaboración propia a partir de la información extraída de los atestados de la GUB.

Figura 4

Hurtos con luz y domicilios

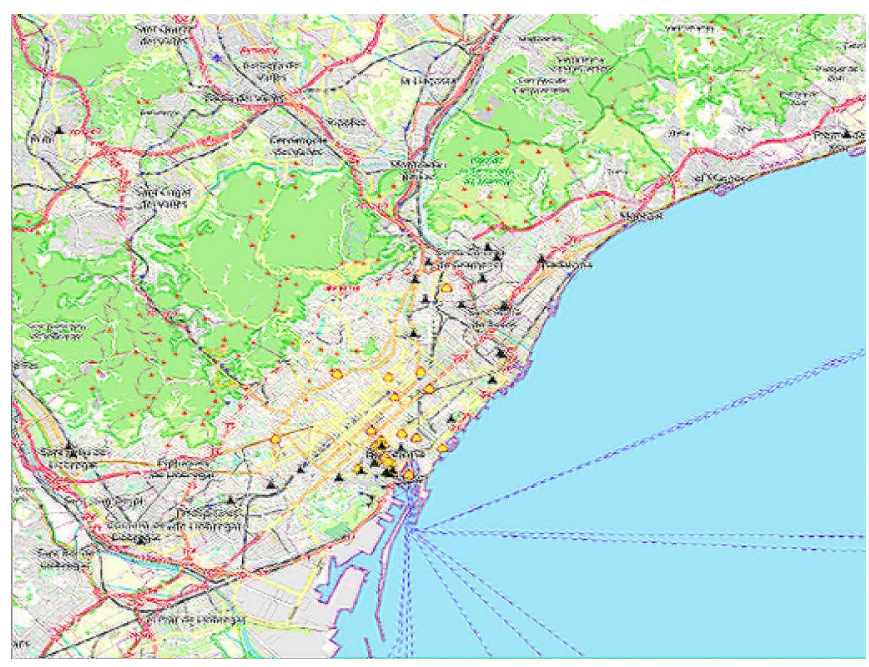

Nota: Los triángulos negros simbolizan el domicilio y los puntos amarillos los hurtos cometidos a la luz del día.

Figura 5

Hurtos sin luz y domicilios

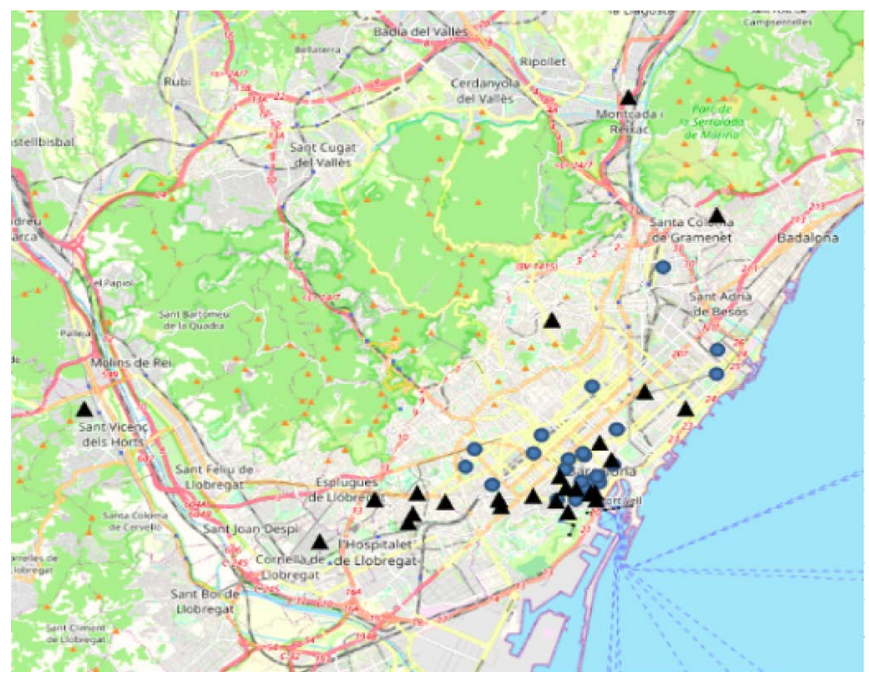

Nota: Los triángulos negros simbolizan el domicilio y los puntos azules los hurtos cometidos durante la noche.
La mayoría de ladrones delinquen en una zona turística y muchos de ellos recorren una distancia corta, sin que exista una diferencia estadísticamente significativa (tabla 5).

Tabla 5

Tipo de distancia recorrida (corta o larga) relacionada con el sitio (turístico o no).

\begin{tabular}{ccccc}
$\mathbf{n}=\mathbf{6 6}$ & No turístico & Turístico & $\mathbf{n}$ & $\mathbf{P}$ \\
\hline Corta & 10 & 32 & 42 & $\mathrm{p}=0.289$ \\
Larga & 8 & 18 & 24 & \\
Total & 18 & 48 & 66 & \\
\hline
\end{tabular}

Nota: Elaboración propia a partir de la información extraída de los atestados de la GUB.

Los hurtadores que recorren distancias cortas actúan entre semana $y$, en cambio, los que recorren distancias más largas roban durante el fin de semana $\left(X^{2}(1)=4.737\right.$; $\mathrm{p}=0.028 ; \mathrm{V}=0.030$ ) (tabla 6 y figuras $6-7$ ). La diferencia es estadísticamente significativa (tabla 6).

Tabla 6

Tipo de distancia (corta/larga) en relación con la parte de la semana (entre semana/ fin de semana).

$\begin{array}{ccccc}\mathbf{N}=\mathbf{6 6} & \text { Entre semana } & \text { Fin de semana } & \mathrm{n} & \mathrm{p} \\ \text { Corta } & 29 & 13 & 42 & \mathrm{p}=0.028 \\ \text { Larga } & 10 & 14 & 24 & \\ \text { Total } & 39 & 27 & 66 & \end{array}$

Nota: Elaboración propia a partir de la información extraída de los atestados de la GUB.

Figura 6

Hurtos entre semana y domicilios

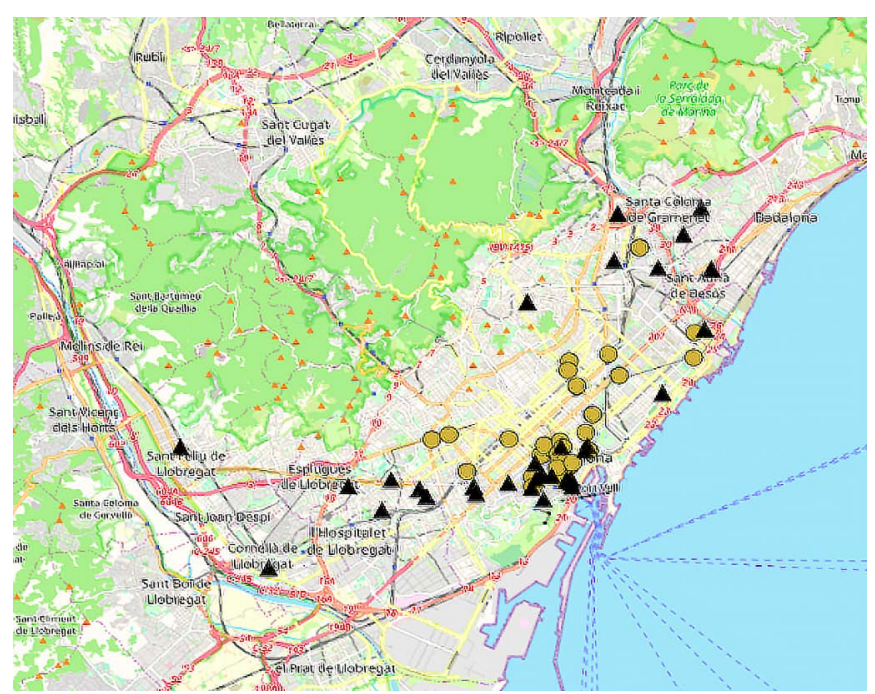

Nota: Los triángulos negros simbolizan el domicilio y los puntos verdes los hurtos cometidos entre semana. 
Figura 7

Hurtos fin de semana y domicilios

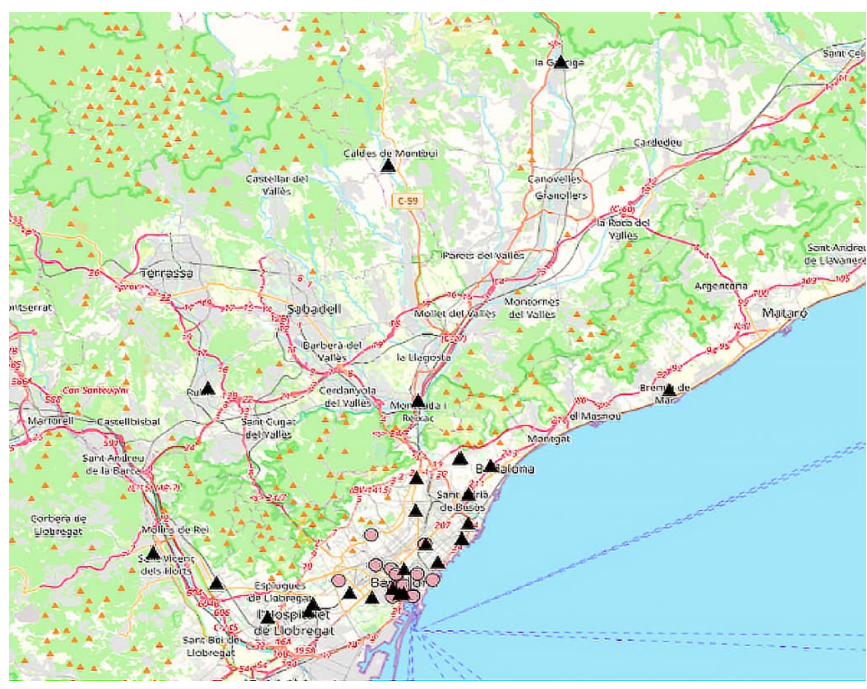

Nota: Los triángulos negros simbolizan el domicilio y los puntos rosa los hurtos cometidos durante el fin de semana.

\section{Antecedentes}

Análisis descriptivo de los antecedentes

Los 15 ladrones (con 4 antecedentes o más) roban en el centro de Barcelona y viven también en la misma zona (figura 8).

\section{Figura 8}

Hurtos principales, antecedentes y domicilio

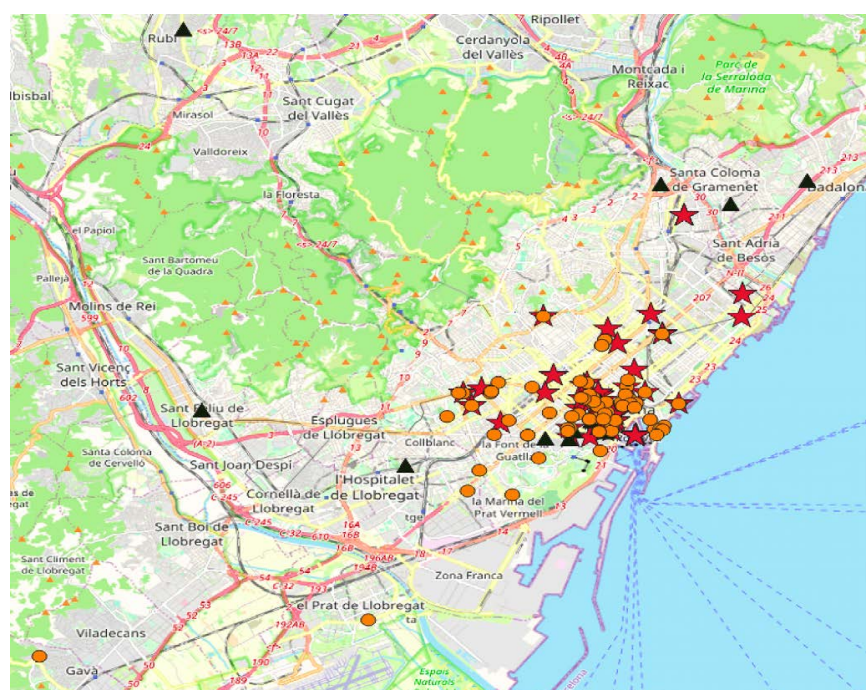

Nota: Los puntos naranjas representan los antecedentes, las estrellas rojas los hurtos

$\mathrm{n}=15$ y los triángulos negros los domicilios de los hurtadores.

La mayoría de los 15 hurtadores seleccionados con 4 antecedentes o más se desplazan hacia al centro para hur- tar, ya que la mayoría de hurtos se cometen en el centro de la ciudad, y aunque algunos de ellos viajan para hurtar desde más lejos, la mayoría se desplaza en dirección al centro (figura 9).

Figura 9

Direccionalidad de los hurtos

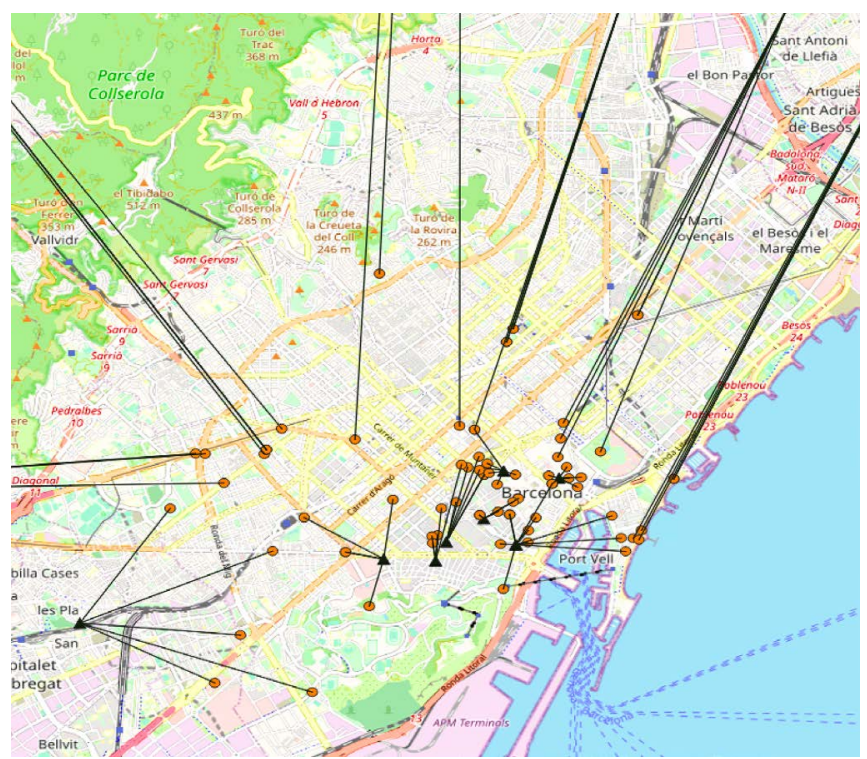

Nota: Los puntos naranjas representan los antecedentes, los triángulos negros los domicilios de los hurtadores y las líneas negras las direcciones y distancias recorridas.

La mayoría de hurtadores actúan en una zona, dentro de un rango determinado (figura 10).

Figura 10

Zonas donde actúan los hurtadores

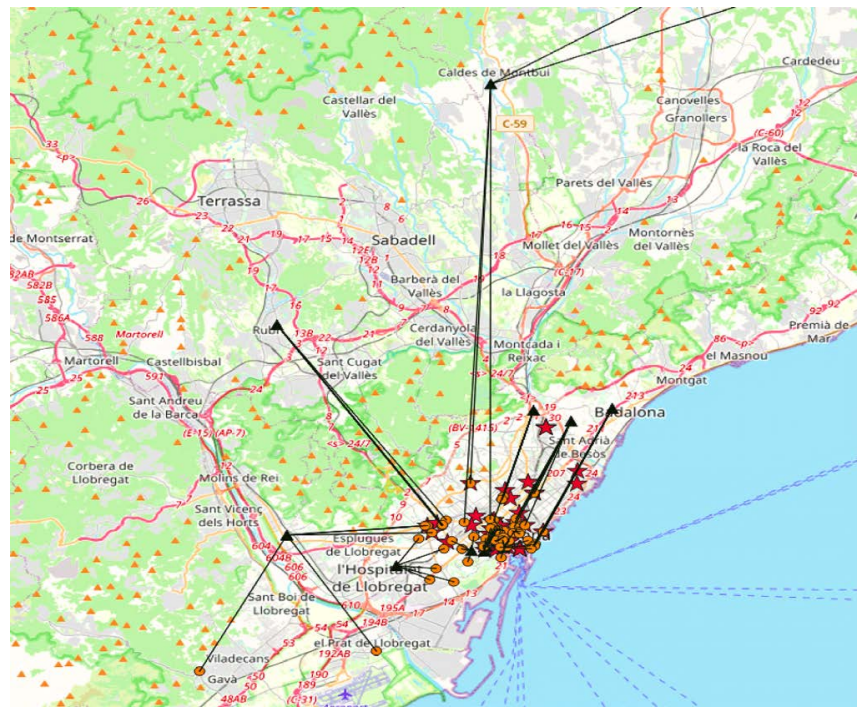

Nota: Los puntos naranjas representan los antecedentes, los triángulos negros los domicilios y las líneas negras las direcciones recorridas. 
Los 15 ladrones seleccionados según el número de antecedentes se dividen equitativamente entre commuters y marauders (tabla 7). La mayoría recorren poca distancia, menos de cinco kilómetros (53.3\%) y hurtan de forma recurrente, dejando pasar poco tiempo entre hurtos $(66.7 \%)$ (tabla 7$)$.

Tabla 7

Características del hurtador

\begin{tabular}{|ccc|}
\hline Variables $(\mathbf{n}=\mathbf{1 5})$ & Frecuencia & Porcentaje \\
\hline Commuter & Tipo hurtador \\
\hline Marauder & 7 & $46.7 \%$ \\
& 8 & $53.3 \%$ \\
\hline $5-5$ & Distancia Recorrida \\
\hline $10-15$ & 8 & $53.3 \%$ \\
$15-20$ & 3 & $20.0 \%$ \\
\hline $20-25$ & 1 & $6.70 \%$ \\
\hline $25-30$ & 1 & $6.70 \%$ \\
& Tipo de distancia recorrida & $6.70 \%$ \\
\hline Corta & 10 & $6.69 \%$ \\
Larga & 5 & $66.7 \%$ \\
& Frecuencia (temporal) & \\
\hline Alta & 10 & $63.3 \%$ \\
Baja & 5 & $33.3 \%$ \\
\hline
\end{tabular}

Nota: Elaboración propia a partir de la información extraída de los atestados de la GUB.

\section{Análisis bivariado de los antecedentes}

Los delincuentes analizados se reparten por igual entre commuters y marauders, y ambos grupos tienden a delinquir de forma recurrente, es decir, sin dejar pasar mucho tiempo entre los hurtos realizados. Concretamente, más de $1 / 3$ de la muestra vuelve a delinquir antes de que hayan pasado 71 días (tabla 8).

Tabla 8

Perfil del delincuente (commuter/marauder) y frecuencia del hurto (alta/baja)

\begin{tabular}{cccc}
$\mathrm{n}=15$ & Alta & Baja & $\mathrm{N}$ \\
\hline Commuter & 6 & 1 & 7 \\
Marauder & 6 & 2 & 8 \\
Total & 12 & 3 & 15 \\
\hline
\end{tabular}

Nota: Elaboración propia a partir de la información extraída de los atestados de la GUB.
La gran mayoría de commuters recorren una distancia larga (por encima de los $8.62 \mathrm{~km}$ ) y todos los marauders recorren distancias cortas, por debajo de los $8.62 \mathrm{~km}$. En conclusión, 2/3 partes de los hurtadores recorren menos de $8.62 \mathrm{~km}$ (tabla 9).

Tabla 9

Tipo distancia (corta/largar) relacionada con el perfil (commuter) marauder)

\begin{tabular}{cccc}
$\mathbf{n = 1 5}$ & Commuter & Marauder & $\mathbf{n}$ \\
Corta & 2 & 8 & 10 \\
Larga & 5 & 0 & 5 \\
Total & 7 & 8 & 15 \\
\hline
\end{tabular}

Nota: Elaboración propia a partir de la información extraída de los atestados de la GUB.

Dentro de las 2/3 partes de la muestra que recorren una distancia corta, la gran mayoría delinque de forma más recurrente, es decir que pasa poco tiempo entre uno y otro robo. El otro 1/3 de los delincuentes recorre distancias largas, también de forma recurrente (tabla 10).

Tabla 10

Distancia recorrida (corta/larga) en relación con la frecuencia (alta/baja).

\begin{tabular}{cccc}
$\mathrm{n}=\mathbf{1 5}$ & Alta & Baja & $\mathrm{N}$ \\
\hline Corta & 8 & 2 & 10 \\
Larga & 4 & 1 & 5 \\
Total & 12 & 3 & 15 \\
\hline
\end{tabular}

Nota: Elaboración propia a partir de la información extraída de los atestados de la GUB.

\section{Análisis de la safety zone}

La safety zone para los marauders es de $0.36 \mathrm{~km}$ y no tiene una direccionalidad privilegiada en la comisión de los hurtos, debido a que se ha obtenido un valor de la pendiente de 0.65 (próximo a 0.5). Esto significa que el domicilio se sitúa en el centro de los antecedentes. Este resultado es significativo, puesto que el coeficiente de correlación que se ha obtenido en la regresión lineal es de 0.98 (figura 11). 
Figura 11

Análisis de la safety zone

Safety zone

$y=0,6467 x+0,3561$

$\mathrm{R}^{2}=0,9836$

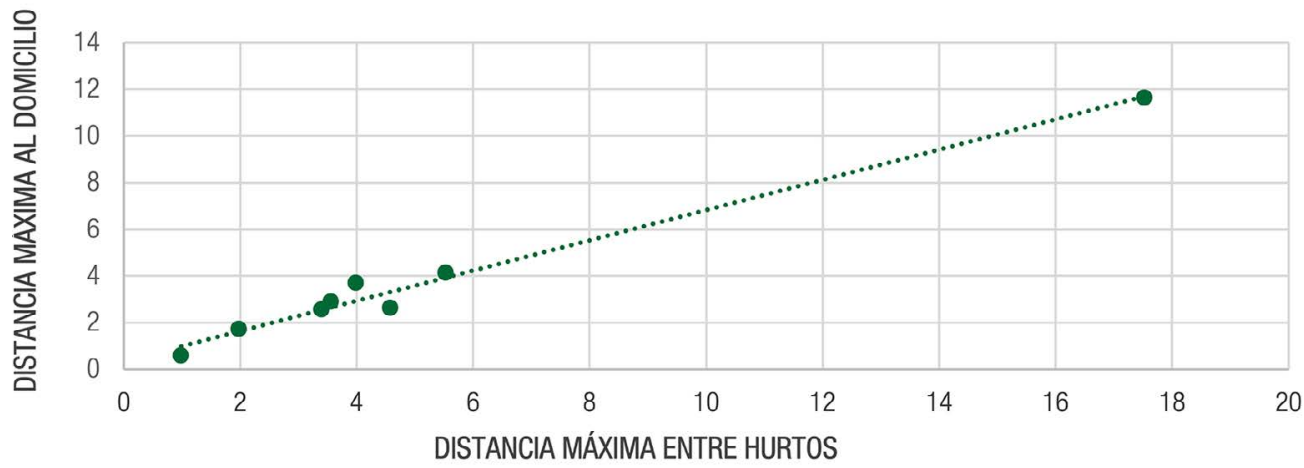

Nota: Los puntos representan los valores hallados para los marauders con antecedentes y la línea la recta de regresión.

\section{Decay distance}

El grupo de los marauders presenta un patrón claro, pues la probabilidad de robo disminuye con respecto a la distancia entre el lugar del robo y el domicilio (figura 12A), contrario a lo que sucede con los commuters (figura 12B) y con el grupo mixto (12C), que no presentan un patrón claro. Para el grupo de los marauders observamos que la mayoría hurta en un radio inferior a $3 \mathrm{~km}(85 \%)$. La mayoría de los commuters hurta en un radio entre 7 y $13 \mathrm{~km}$ (58\%), aunque debido a la dispersión de la muestra no puede considerarse rango de actuación. Para el grupo mixto se encuentra que los delincuentes de la muestra hurtan en una distancia inferior a $7 \mathrm{~km}(59 \%)$, aunque tampoco puede considerarse como rango de actuación, por el mismo motivo anterior.

Figura 12

Análisis de la decay distance

a. Marauders

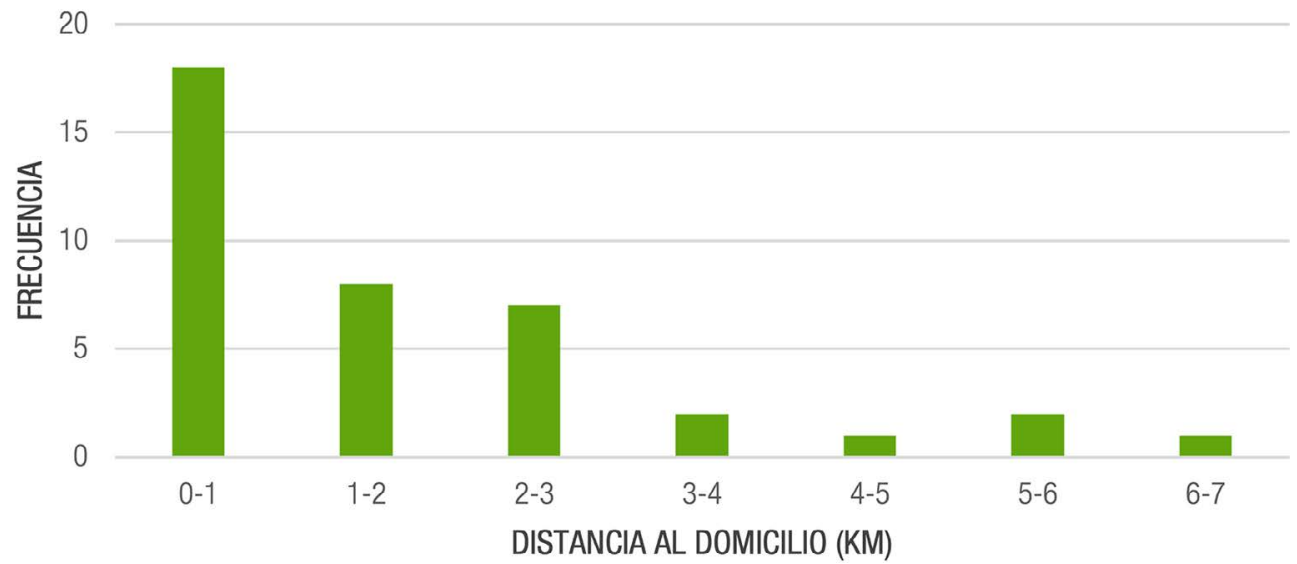

Nota: Elaboración propia a partir de la información extraída de los atestados de la GUB. 
b. Commuters

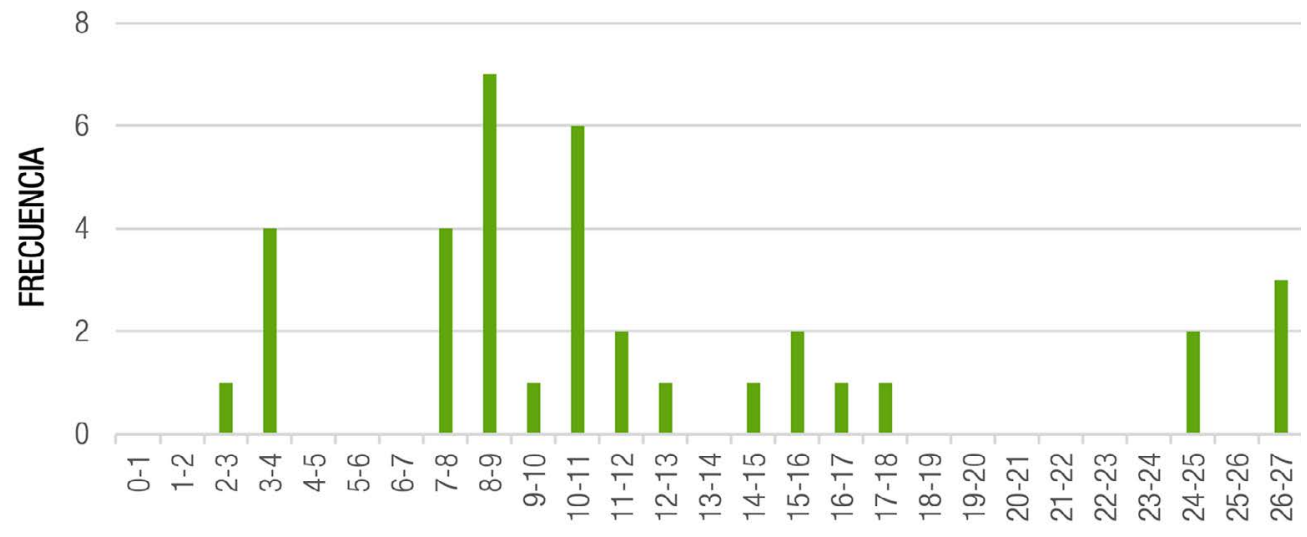

\section{DISTANCIA AL DOMICILIO (KM)}

Nota: Elaboración propia a partir de la información extraída de los atestados de la GUB.

c. Grupo mixto

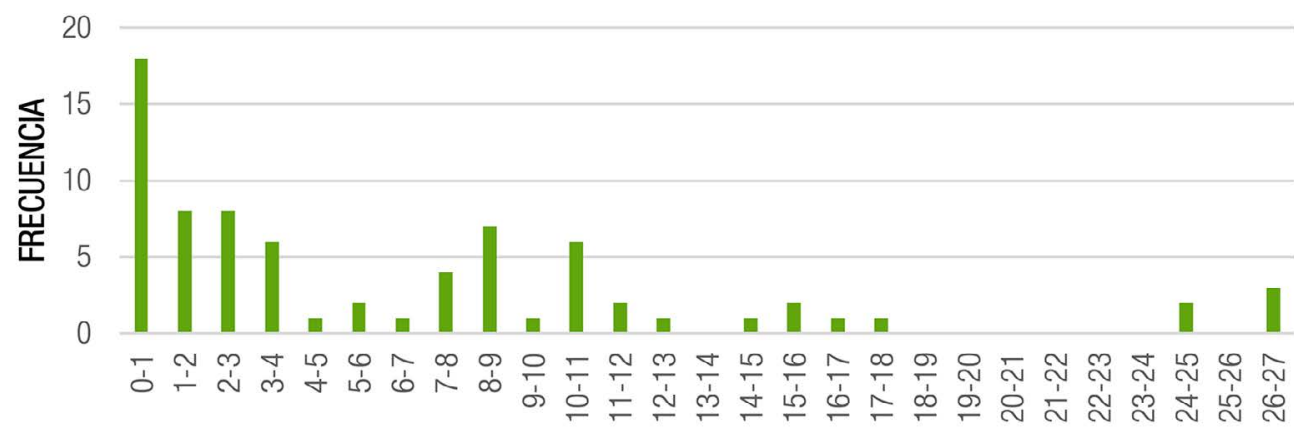

DISTANCIA AL DOMICILIO (KM)

Nota: Elaboración propia a partir de la información extraída de los atestados de la GUB.

Si se modeliza dicho comportamiento en el grupo de los marauders, utilizando una distribución exponencial negativa $y=A e^{-B x}$, se halla que una buena aproximación

Figura 13

Modelo para la decay distance

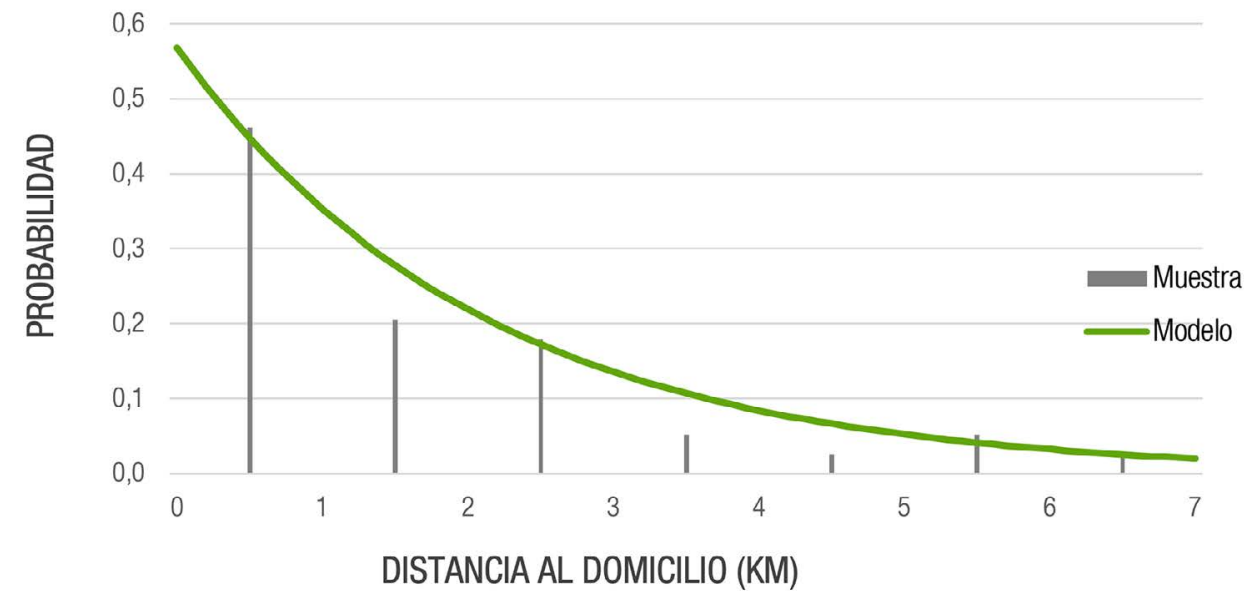

$\left(R^{2}=0.84\right)$ viene dada por los valores $A=0,57$ y $B=0.48$ (figura 13).

Nota: Los valores en azul corresponden a los representados en la figura $12 \mathrm{~A}$, pero en lugar de representar frecuencias se representan probabilidades, es decir, la frecuencia normalizada del total de la muestra. Para los valores en el eje horizontal se toma el valor medio del rango utilizado en la figura $12 \mathrm{~A}$. 


\section{Discusión}

Coincidiendo con la teoría de la geografía humana, los ladrones de Barcelona recorren una mayor distancia cuando actúan de día y menos en la noche, en consonancia con la explicación de que por necesidades biológicas (horas de sueño) y por otras más lógicas, como por no desplazarse y ser descubiertos, estos deciden hurtar a una distancia más próxima al domicilio durante la noche y más lejana durante el día. También se encuentra que en Barcelona la mayoría de delincuentes que roban entre semana recorren distancias cortas, en comparación con las que recorren el fin de semana, que son más largas, probablemente debido al intercambio y a la movilización de personas que se producen durante esos momentos, lo que representa la oportunidad de que se cometan más delitos y facilita la huida del ladrón, concentrando en otras zonas las víctimas potenciales de los días entre semana.

Según la teoría de Brantinghan \& Brantingham (1981), los ladrones viven a una distancia próxima al lugar donde se comete el hurto y, en Barcelona, la mayoría de ellos recorren una distancia de hasta $5 \mathrm{~km}$, según las investigaciones de Rhodes \& Canly (1981), Rengert et al. (1999) y Sarangi \& Youngs (2006).

Sin embargo, quienes hurtan en comercios recorren una distancia más larga que los que no lo hacen, posiblemente por el miedo a ser reconocidos, a diferencia de los que hurtan fuera del comercio, donde hay una gran variedad de víctimas y resulta más difícil su reconocimiento.

Además, los resultados muestran que los hurtadores de Barcelona se dividen entre commuters y marauders, sin que prevalezca un perfil por encima del otro, tal y como expusieron Kocksis \& Irwin (1997); recorren distancias largas los commuters, como en la teoría de Canter \& Larkin (1993), y distancias cortas los marauders, como en la teoría de Daele \& Bernsaco (2012), pero ambos hurtan de forma recurrente, como se observa en los estudios de Goodwill \& Alison (2006) y Bernasco (2008).

En consonancia con la investigación de Canter \& Hodge (2000), también existe una safety zone entre los marauders de Barcelona, concretamente de $0.36 \mathrm{~km}$, distancia en la que, como dice la teoría, no delinquen por miedo a ser reconocidos. Además, como se señala en Kocksis \& Irwin (1997), se ha comprobado que no existe una direccionalidad privilegiada para la comisión del hurto entre estos marauders, ya que el domicilio se halla centrado respeto los hurtos cometidos. Finalmente, se comprobó que los marauders mantienen una decay distance, como lo señalan Emeno \& Benell (2013) y Block \& Bernasco (2009), confirmando que la probabilidad de hurto disminuye a medida que los delincuentes se alejan del domicilio (Bernasco \& Kooistra, 2010; Kocsis \& Irwin, 1997). No se aplica esta conclusión respecto de los commuters, tal y como indican Block \& Bernasco (2009).

\section{Conclusiones}

Los hurtos en Barcelona son una problemática actual, no solo por su incremento, sino también por su influencia en la percepción de inseguridad ciudadana. Por ello, este estudio realizó un perfil geográfico, fundamental para la prevención del hurto, la delimitación de recursos y la disposición de esfuerzos policiales para reducir su incidencia en la ciudad.

En relación con la distancia recorrida por el delincuente al lugar de hurto, la mayoría de los atracadores reside en la zona metropolitana de Barcelona, cerca del lugar donde cometen el delito (a menos de $5 \mathrm{~km}$ la mayoría de ellos). Se desplazan hacia al centro para robar, y aunque existen diferentes distancias, predominan las distancias cortas. No obstante, los ladrones que recorren distancias cortas tienden a delinquir por fuera del comercio, contrario de los que recorren distancias largas. Además, los que recorren distancias cortas tienden a delinquir de noche, a diferencia de los que recorren largas distancias, que actúan de día. Estos últimos roban durante el fin de semana y los primeros, que recorren distancias cortas, hurtan entre semana.

Referente al perfil criminal, los hurtadores de Barcelona se dividen en commuters y marauders equitativamente. Los primeros recorren distancias más largas (más de $8 \mathrm{~km}$ ) que los otros. Ambos tipos delinquen de forma recurrente, en intervalos de tiempo cortos (antes de 70 días). Sin embargo, los que recorren distancias más cortas roban con mayor frecuencia que los que recorren distancias largas.

Por último, en consideración con la safety zone y la decay distance cabe remarcar que los marauders no presentan una dirección privilegiada para hurtar, desplazándose hacia cualquier dirección, partiendo de un punto central (lugar de residencia) y respetando una zona de $0.36 \mathrm{~km}$ en la cual no hurtan. En consecuencia, en distancias mayores a $6 \mathrm{~km}$ la probabilidad de los delitos se reduce drásticamente para este perfil. No obstante, este patrón no se mantiene en el caso de los commuters.

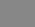

西 
Las principales limitaciones de esta investigación giran en torno a la muestra, debido a que, por la pandemia, no ha sido posible recoger un mayor número de casos, aparte de los que constan en la investigación realizada por los agentes de la GUB durante la primera semana de marzo, lo que significa que, probablemente, esté sesgada.

Otra limitación es que esta muestra solo cuenta con los casos recogidos por los agentes de GUB, pero también hay un gran número de casos que no han sido denunciados, o no han sido registrados por los agentes y no hacen parte de la muestra, aunque probablemente influyan en los resultados en caso de que se recojan más casos en zonas donde ya existe una mayor vigilancia policial.

En futuros estudios se deberían abarcar todos los factores de la muestra del perfil geográfico, junto con otros datos del perfil del hurtador, y realizar un análisis aplicando regresión logística, a fin de permitir al investigador y a la Policía conocer qué factores influyen en la comisión del hurto, para poder tratarlos con el objeto de prevenir y reducir la comisión del delito. No obstante, para poder llevarlos a cabo y que sean representativos sería necesario trabajar con más muestras.

Finalmente, atendiendo a las teorías de Hägerstrand (1970), Cohen \& Felson (1979), Cornish \& Clarke (1986), Canter \& Larkin (1993), Rossmo (1995), Canter \& Hodge (2000) y Brantingham \& Brantingham (2008), se recomiendan medidas de prevención espacial específicas, junto con una mayor capacidad de recursos de vigilancia en las zonas turísticas, comercios y, sobre todo, en el distrito de Ciutat Vella, en horas de la tarde, especialmente a las 17:00 horas.

Además, teniendo en cuenta el perímetro de residencia de los hurtadores, se recomienda establecer controles en Ciutat Vella, particularmente entre semana, cuando las distancias recorridas por los delincuentes son más cortas, sobre todo a partir de las 17:00 horas. También se considera necesario que, durante el fin de semana, cuando los ladrones recorren distancias más largas, los controles se realicen en las entradas y salidas de la ciudad durante el día, para poder realizar registros e identificar a los delincuentes.

Del mismo modo puede realizarse un control sobre la reincidencia en los hurtos, teniendo en cuenta que la mayoría de ladrones vuelve a delinquir en los 70 días posteriores a la comisión del hurto. Se considera oportuno crear un registro de los delincuentes, que serviría para controlar tanto los accesos de Barcelona como las entradas y salidas de los transportes públicos para, de esta manera, conocer hacia dónde se desplazan o si salen de la ciudad. Esto hace posible realizar un seguimiento de la actividad de los ladrones y, dado que se conocen su domicilio y sus antecedentes, es más fácil clasificarlo como marauder, lo que implica que tiene una probabilidad elevada de volver a hurtar dentro de los próximos 70 días a la comisión del robo, en un radio inferior a $3 \mathrm{~km}$ del domicilio; en el caso de ser clasificado como commuter resulta más complicada su perfilación. Con esta información, resulta posible trazar zonas donde es necesario establecer más vigilancia y otras donde no resulte tan necesario. Gran parte de la información que se ha extraído de esta investigación puede servir de ayuda a la Policía para localizar el domicilio del ladrón, pues se ha identificado que entre semana se hurta más de noche y que los delincuentes nocturnos acostumbran a recorrer distancias cortas, contrario a los sujetos que roban en los fines de semana, que recorren distancias largas y delinquen durante el día. Gracias a esto, se tiene información más clara sobre dónde empezar a buscar, además de características puntuales para la clasificación dentro de alguno de los perfiles señalados. En el caso de marauders, si se desconoce su domicilio, podríamos llegar a deducirlo si tuviéramos información sobre ciertas características del hurto. Una vez clasificado, se puede en consecuencia establecer un radio de búsqueda en el que probablemente resida.

\section{Conflicto de intereses}

Los autores manifiestan no tener ningún conflicto de intereses.

\section{Referencias}

Bernasco, W. (2007). The usefulness of measuring spatial opportunity structures for tracking down offenders: A theoretical analysis of geographic offender profiling using simulation studies. Psychology, Crime \& Law, 13(2), 155-171. https://doi. org/10.1080/10683160600558402

Bernasco, W. (2008). Them again? Same-offender involvement in repeat and near repeat burglaries. European Journal of Criminology, 5(4), 411-431. https://doi. org/10.1177/1477370808095124

Bernasco, W., \& Kooistra, T. (2010). Effects of residential history on commercial robbers' crime location choi- 
ces. European Journal of Criminology, 7(4), 251-265. https://doi.org/10.1177/1477370810363372

Bernasco, W., \& Nieuwbeerta, P. (2005). How do residential burglars select target areas? A new approach to the analysis of criminal location choice. British Journal of Criminology, 45(3), 296-315. https://doi. org/102013.1093/bjc/azh070

Bernasco, W., Block, R., \& Ruiter, S. (2013). Go where the money is: Modeling street robbers' location choices. Journal of Economic Geography, 13(1), 119-143. https://doi.org/10.1093/jeg/lbs005

Bernasco, W., Johnson, S. D., \& Ruiter, S. (2015). Learning where to offend: Effects of past on future burglary locations. Applied Geography, 60, 120-129. https://doi. org/10.1016/j.apgeog.2015.03.014

Bernasco, W., Ruiter, S., \& Block, R. (2017). Do street robbery location choices vary over time of day or day of week? A test in Chicago. Journal of Research in Crime and Delinquency, 54(2), 244-275. https://doi. org/10.1177/0022427816680681

Block, R., \& Bernasco, W. (2009). Finding a serial burglar's home using distance decay and conditional origin-destination patterns: a test of empirical Bayes journey-to-crime estimation in the Hague. Journal of Investigative Psychology and Offender Profiling, 6(3), 187-211. https://doi.org/10.1002/jip.108

Boivin, R. (2018). Routine activity, population (s) and crime: Spatial heterogeneity and conflicting propositions about the neighborhood crime-population link. Applied geography, 95, 79-87. https://doi. org/10.1016/j.apgeog.2018.04.016

Bowers, K., \& Johnson, S. D. (2015). Poetry in motion: the case of insider and outsider offenders. En M. A. Andresen et al. (Eds.), The Criminal Act (pp. 115-130). Palgrave Macmillan. https://doi. org/10.1057/9781137391322_9

Bowers, K. J., \& Johnson, S. D. (2016). Situational prevention. En D. Weisburd, D. Farrington, C. Gill (Eds.), What Works in Crime Prevention and Rehabilitation: Lessons from systematic reviews (pp. 111-135). Springer. https://doi.org/10.1007/978-1-4939-3477-5_4

Branic, N. (2015). Routine activities theory. En W. G. Jennings (Ed.), Encyclopedia of Crime and Punish- ment (pp.1-3). John Wiley \& Sons, Inc. https://doi. org/10.1002/9781118519639.wbecpx059

Brantingham, P. J., \& Brantingham, P. L. (1981). Notes of the geometry of crime. En P. J. Brantingham., \& P. L. Brantingham (Eds.), Environmental Criminology (pp. 27-54). Sage Publications.

Brantingham, P. J., \& Brantingham, P. L. (2008). Crime pattern theory. En R. Wortley y M. Townsley (Eds.), Environmental Criminology and Crime Analysis (pp. 7893). Willan.

Brunt, P., \& Hambly, Z. (1999). Tourism and crime: A research agenda. Crime Prevention and Community Safety, 1(2), 25-36. https://doi.org/10.1057/palgrave. cpcs.8140013

Canter, D. \& Hodge, S. (2000). Criminals mental maps. En L. S. Turnball, E. Hallisey-Hendrix \& B. D. Dent (Eds.), Atlas of Crime (pp.187-191). Oryx Press.

Canter, D., \& Larkin, P. (1993). The environmental range of serial rapists. Journal of Environmental Psychology, 13(1), 63-69. https://doi.org/10.1016/ S0272-4944(05)80215-4

Castro, M. C., \& Martini, H. A. (2014). Potencia estadística y cálculo del tamaño del efecto en $\mathrm{G}^{*}$ Power: complementos a las pruebas de significación estadística y su aplicación en psicología. Salud \& Sociedad, 5(2), 210-224.

Cohen, L. E., \& Felson, M. (1979). Social change and crime rate trends: A routine activity approach. American Sociological Review, 44(4), 588-608. https://doi. org/10.2307/2094589

Cornish, D. B., \& Clarke, R. V. (1986). The reasoning criminal: Rational choice perspectives on offending. Springer-Veralg.

Daele, S. V., \& Bernasco, W. (2012). Exploring directional consistency in offending: The case of residential burglary in The Hague. Journal of Investigative Psychology and Offender Profiling, 9(2), 135-148. https://doi. org/10.1002/jip.1358

Eck, J. E. (2018). Regulation for high-crime places: Theory, evidence, and principles. The Annals of the American Academy of Political and Social Science, 679(1), 106120. https://doi.org/10.1177/0002716218778764 
Emeno, K., \& Bennell, C. (2013). The effectiveness of calibrated versus default distance decay functions for geographic profiling: a preliminary examination of crime type. Psychology, Crime \& Law, 19(3), 215-232. https://doi.org/10.1080/1068316X.2011.621426

Felson, M., \& Boivin, R. (2015). Daily crime flows within a city. Crime Science, 4(1), 1-10. https://doi. org/10.1186/s40163-015-0039-0

Frith, M. J., Johnson, S. D., \& Fry, H. M. (2017). Role of the street network in burglars' spatial decisionmaking. Criminology, 55(2), 344-376. https://doi. org/10.1111/1745-9125.12133

Glasner, P., \& Leitner, M. (2017). Evaluating the impact the weekday has on near-repeat victimization: A spatio-temporal analysis of street robberies in the city of Vienna, Austria. ISPRS International Journal of Geo-Information, 6(1), 3-21. https://doi.org/10.3390/ ijgi6010003

Goodwill, A. M., \& Alison, L. J. (2006). The development of a filter model for prioritising suspects in burglary offences. Psychology, Crime \& Law, 12(4), 395-416 https://doi.org/10.1080/10683160500056945

Haberman, C. P., \& Ratcliffe, J. H. (2015). Testing for temporally differentiated relationships among potentially criminogenic places and census block street robbery counts. Criminology, 53(3), 457-483. https://doi. org/10.1111/1745-9125.12076

Hägerstrand, T. (1989). Reflections on "what about people in regional science?". Papers of the Regional Science Association, 66(1), 1-6. https://doi. org/10.1111/j.1435-5597.1989.tb01166.x

Hart, T. C., \& Miethe, T. D. (2014). Street robbery and public bus stops: a case study of activity nodes and situational risk. Security Journal, 27(2), 180-193. https:// doi.org/10.1057/sj.2014.5

Hipp, J. R., \& Kim, Y. A. (2019). Explaining the temporal and spatial dimensions of robbery: Differences across measures of the physical and social environment. Journal of Criminal Justice, 60, 1-12. https://doi. org/10.1016/j.jcrimjus.2018.10.005

Jefatura del Estado Español (1995). Ley Orgánica 10 de 23 de noviembre. Código Penal. BOE-A-1995-25444. https://www.boe.es/eli/es/lo/1995/11/23/10/con
Kocsis, R. N., \& Irwin, H. J. (1997). An analysis of spatial patterns in serial rape, arson, and burglary: the utility of the circle theory of environmental range for psychological profiling. Psychiatry, Psychology \& Law, 4(2), 195206 https://doi.org/10.1080/13218719709524910

Lantz, B., \& Hutchison, R. (2015). Co-offender ties and the criminal career: group characteristics, persistence, desistance, and the individual offender. Journal of Research in Crime and Delinquency, 52(5), 658-690. 690.https://doi.org/10.1177/0022427815576754

Lantz, B., \& Ruback, R. B. (2017). A networked boost: Burglary co-offending and repeat victimization using a network approach. Crime \& Delinquency, 63(9), 10661090.https://doi.org/10.1177/0011128715597695

Long, D., Liu, L., Feng, J., Zhou, S., \& Jing, F. (2018). Assessing the influence of prior on subsequent street robbery location choices: a case study in ZG city, China. Sustainability, 10(6), 1818-1834. https://doi. org/10.3390/su10061818

Monk, K., Heinonen, J. A., \& Eck, J. E. (2010). Street Robbery: The Problem of Street Robbery What This Guide Does and Does Not Cover. Center for Problem-Oriented Policing. U.S. Department of Justice https://cops. usdoj.gov/RIC/Publications/cops-p181-pub.pdf

Pettiway, L. E. (1982). Mobility of robbery and burglary offenders: Ghetto and nonghetto spaces. Urban Affairs Quarterly, 18(2), 255-270. https://doi. org/10.1177/004208168201800206

Phillips, P. D. (1980). Characteristics and Typology of the Journey to Crime. En D. Georges-Abeyie y K. D. Harries (Eds.), Crime: A spatial perspective (pp. 167-180). Columbia University Press.

Piza, E. L. \& Kennedy, D. (2003). Transit stops, robbery, and routine activities: Examining street robbery in the Newark, NJ subway environment. https://proceedings. esri.com/library/userconf/proc04/docs/pap1303.pdf

Rengert, G. F., Piquero, A. R., \& Jones, P. R. (1999). Distance decoy re-examined. Criminology, 37(2), 425-427. https://doi.org/10.1111/j.1745-9125.1999.tb00492.x

Rhodes, W. M., \& Canly, C. (1981). Crime and mobility: An empirical study. En P. J. Brantinghman \& P. L. Brantingham (Eds.), Environmental Criminology (pp. 167188). Sage Publications. 
Rossmo, D. K. (1995). Geographic profiling: Target patterns of serial murderers [Tesis doctoral, School of Criminology, Simon Fraser University]. https://core.ac.uk/ reader $/ 56371040$

Sarangi, S., \& Youngs, D. (2006). Spatial patterns of Indian serial burglars with relevance to geographical profiling. Journal of Investigative Psychology and Offender Profiling, 3(2), 105-115. https://doi.org/10.1002/jip.38

Sidebottom, A., \& Wortley, R. (2016). Environmental criminology. En A. R. Piquero (Ed.), The Handbook of Criminological Theory (pp. 156-181). John Wiley \& Sons, Inc. https://doi.org/10.1002/9781118512449.ch9

Tompson, L., \& Bowers (2013). A stab in the dark? A research note on temporal patterns of Street robbery. Journal of Research in Crime and Delinquency, 50(4), 616-631. https://doi.org/10.1177/0022427812469114
Tucker, R., O'Brien, D. T., Ciomek, A., Castro, E., Wang, Q., \& Phillips, N. E. (2021). Who 'Tweets' Where and When, and How Does it Help Understand Crime Rates at Places? Measuring the Presence of Tourists and Commuters in Ambient Populations. Journal of Quantitative Criminology, 1-2. https://doi.org/10.1007/ s10940-020-09487-1

Vandeviver, C., \& Bernasco, W. (2019). "Location, location, location": Effects of neighborhood and house attributes on burglars' target selection. Journal of Quantitative Criminology, 36, 779-821. https://doi. org/10.1007/s10940-019-09431-y

Weisburd, D. (2015). The law of crime concentration and the criminology of place. Criminology, 53(2), 133157. https://doi.org/10.1111/1745-9125.12070 